\title{
NILPOTENT CONTROL SYSTEMS
}

\author{
Elisabeth REMM and Michel GOZE
}

\begin{abstract}
We study the class of matrix controlled systems associated to graded filiform nilpotent Lie algebras. This generalizes the nonlinear system corresponding to the control of the trails pulled by car.
\end{abstract}

\section{Introduction}

When we consider the problem of a mobile robot on the plane, then the front wheels of the driving car are subjected to two controls (driving and turning speed). If the driving car pulls a chain of $n$ trailers, then a model for the kinematic behavior of this system is given by :

$$
\text { (1) } \quad\left\{\begin{array}{l}
\dot{x_{1}}=u_{1} \\
\dot{x}_{2}=u_{2} \\
\dot{x}_{3}=x_{2} u_{1} \\
\dot{x}_{4}=x_{3} u_{1} \\
\vdots \\
\bullet \\
\dot{x}_{n}=x_{n-1} u_{1}
\end{array}\right.
$$

where $u_{1}$ and $u_{2}$ are the control functions. This system can be written in the "canonical form":

$$
\left.\dot{X}(t)=\left[u_{1}(t) A_{1}+u_{2}(t) A_{2}\right)\right] X(t)
$$

2000 Mathematics Subject Classification: 17B30, 93B25, 22F99, 34K35.

Servicio de Publicaciones. Universidad Complutense. Madrid, 2002 
where $A_{1}$ and $A_{2}$ are the matrices

$$
A_{1}=\left(\begin{array}{ccccc}
0 & & & & \\
0 & 0 & & & \\
0 & 1 & 0 & & \\
\vdots & \ddots & \ddots & \ddots & \\
0 & \cdots & 0 & 1 & 0
\end{array}\right) ; A_{2}=\left(\begin{array}{ccccc}
0 & & & & \\
1 & 0 & & & \\
0 & 0 & 0 & & \\
\vdots & \ddots & \ddots & \ddots & \\
0 & \cdots & 0 & 0 & 0
\end{array}\right)
$$

and $X(t)$ is defined by

$$
X(t)=\left(\begin{array}{llllllll}
1 & & & & & & \\
x_{2}(t) & 1 & & & & & \\
x_{3}(t) & x_{1}(t) & 1 & & & & \\
x_{4}(t) & \frac{1}{2} x_{1}^{2}(t) & x_{1}(t) & \ddots & & & \\
\vdots & \vdots & \vdots & \ddots & \ddots & & \\
\vdots & \vdots & \vdots & \vdots & x_{1}(t) & \ddots & \\
x_{n}(t) & \frac{1}{(n-2) !} x_{1}^{n-2}(t) & \cdots & \cdots & \frac{1}{2} x_{1}^{2}(t) & x_{1}(t) & 1
\end{array}\right)
$$

We can see that the matrices $A_{1}$ and $A_{2}$ generate a $n$-dimensional nilpotent linear Lie algebra which is isomorphic to the filiform Lie algebra $\mathcal{L}_{n}([\mathrm{G} . \mathrm{K}])$, whose brackets are given by:

$$
\left[X_{1}, X_{i}\right]=X_{i+1}
$$

$i=2, \ldots, n-1$, the non-defined brackets being equal to zero or obtained by antisymmetry. The corresponding matrix representation of $\mathcal{L}_{n}$ is :

$$
\left(\begin{array}{llllll}
0 & \cdots & \cdots & \cdots & \cdots & 0 \\
a_{2} & 0 & & & & \vdots \\
a_{3} & a_{1} & 0 & & & \vdots \\
a_{4} & 0 & a_{1} & \ddots & & \vdots \\
\vdots & \vdots & \ddots & & 0 & \vdots \\
a_{n} & 0 & \cdots & 0 & a_{1} & 0
\end{array}\right)
$$

This matrix is the image of an element $\sum a_{i} X_{i}$ for the given faithful representation. 
Remark. The writing of the previous non linear system is possible because we can use a nilpotent minimal representation of the Lie algebra $\mathcal{L}_{n}$. Note that, for a general nilpotent Lie algebra, there does not exist a procedure to determine the minimal possible degree of a faithful representation.

The aim of this work is to generalize to a class of nilpotent Lie algebras, including $\mathcal{L}_{n}$, the corresponding control systems.

\section{Filiform nilpotent Lie algebras}

\subsection{Filiform nilpotent Lie algebras}

Let $\mathcal{G}$ be a $n$-dimensional (real) Lie algebra. Let $\mathcal{C}^{i} \mathcal{G}$ be the characteristic ideal defined by

$$
\left\{\begin{array}{l}
\mathcal{C}^{0} \mathcal{G}=\mathcal{G} \\
\mathcal{C}^{1} \mathcal{G}=[\mathcal{G}, \mathcal{G}] \\
\vdots \\
\mathcal{C}^{i} \mathcal{G}=\left[C^{i-1} \mathcal{G}, \mathcal{G}\right], \quad i \geq 1
\end{array}\right.
$$

The Lie algebra $\mathfrak{g}$ is nilpotent if there is an integer $k$ such that

$$
\mathcal{C}^{k} \mathcal{G}=\{0\}
$$

Definition 1. The $n$-dimensional nilpotent Lie algebra $\mathcal{G}$ is called filiform if the smallest $k$ such that $\mathcal{C}^{k} \mathcal{G}=\{0\}$ is equal to $n-1$.

In this case the descending sequence is

$$
\mathcal{G} \supset C^{1} \mathcal{G} \supset \cdots \supset \mathcal{C}^{n-2} \mathcal{G} \supset\{0\}=\mathcal{C}^{n-1} \mathcal{G}
$$

and we have

$$
\left\{\begin{array}{l}
\operatorname{dim} \mathcal{C}^{1} \mathcal{G}=n-2, \\
\operatorname{dim} \mathcal{C}^{i} \mathcal{G}=n-i-1, \quad i=1, \ldots, n-1
\end{array}\right.
$$

\section{Examples.}

1) The Lie algebra $\mathcal{L}_{n}$ is filiform. 
2) The following $n$-dimensional ( $n$-even) Lie algebra $\mathcal{Q}_{n}$ defined by

$$
\left\{\begin{array}{lll}
{\left[X_{1}, X_{2}\right]=X_{3}} & , & {\left[X_{2}, X_{n-1}\right]=2 X_{n}} \\
\vdots & , & {\left[X_{3}, X_{n-2}\right]=-2 X_{n}} \\
{\left[X_{1}, X_{n-2}\right]=X_{n-1}} & , & \vdots \\
{\left[X_{1}, X_{n-1}\right]=X_{n}} & , & {\left[X_{p}, X_{p+1}\right]=(-1)^{p} 2 X_{n}, \quad p=\frac{n}{2} .}
\end{array}\right.
$$

is filiform.

For this algebra, we have the following linear representation :

$$
\left(\begin{array}{llllllll}
0 & 0 & \cdots & \cdots & \cdots & \cdots & \cdots & 0 \\
a_{2} & 0 & \ddots & & & & & \vdots \\
a_{3} & a_{1} & \ddots & \ddots & & 0 & & \vdots \\
\vdots & 0 & a_{1} & \ddots & \ddots & & & \vdots \\
a_{i} & \vdots & \ddots & \ddots & \ddots & \ddots & & \vdots \\
\vdots & \vdots & 0 & \ddots & \ddots & \ddots & \ddots & \vdots \\
a_{n-1} & 0 & \cdots & \cdots & 0 & a_{1} & 0 & \vdots \\
a_{n} & -a_{n-1} & \cdots & (-1)^{i} a_{i} & \cdots & -a_{3} & a_{1}+a_{2} & 0
\end{array}\right)
$$

\subsection{Graded filiform Lie algebras}

Let $\mathcal{G}$ be a filiform Lie algebra. It is naturally filtered by the ideals $\mathcal{C}^{i} \mathcal{G}$ of the descending sequence. Then we can associate to the filiform Lie algebra $\mathcal{G}$ a graded Lie algebra, noted $g r \mathcal{G}$, which is also filiform. This algebra is defined by

$$
g r \mathcal{G}=\oplus_{i=0, \ldots, n-1} \frac{\mathcal{C}^{i} \mathcal{G}}{\mathcal{C}^{i+1} \mathcal{G}}
$$

We denote $\frac{\mathcal{C}^{i} \mathcal{G}}{\mathcal{C}^{i+1} \mathcal{G}}$ by $\mathcal{G}_{i+1}$. Then we have

$$
g r \mathcal{G}=\mathcal{G}_{1} \oplus \mathcal{G}_{2} \oplus \ldots \oplus \mathcal{G}_{n}
$$

with $\operatorname{dim} \mathcal{G}_{1}=2, \operatorname{dim} \mathcal{G}_{i}=1$ for $2 \leq i \leq n$ and

$$
\left[\mathcal{G}_{i}, \mathcal{G}_{j}\right] \subset \mathcal{G}_{i+j} \quad i+j \leq n
$$


Lemma 1. There is a homogeneous basis $\left\{X_{1}, X_{2}, \ldots, X_{n}\right\}$ of grG such that

$$
\begin{aligned}
X_{1}, X_{2} & \in \mathcal{G}_{1}, \quad X_{i} \in \mathcal{G}_{i} \quad i=2, \ldots, n \\
{\left[X_{1}, X_{i}\right] } & =X_{i+1} \quad i=2, \ldots, n \\
{\left[X_{i}, X_{j}\right] } & =0 \quad 2 \leq i<j \quad i+j \neq n \\
{\left[X_{i}, X_{n-i}\right] } & =(-1)^{i} \alpha X_{n}
\end{aligned}
$$

with $\alpha \in \mathbb{R}$ and $\alpha=0$ if $n$ is even.

A Lie algebra $\mathcal{G}$ is called graded if it is isomorphic to its associated graded Lie algebra :

$$
\mathcal{G}=\operatorname{gr} \mathcal{G}
$$

The classification of graded filiform Lie algebras is described by the following theorem :

Theorem 1. (V) If $n$ is odd, then there are only, up to isomorphism, two $n$-dimensional graded filiform Lie algebras: $\mathcal{L}_{n}$ and $\mathcal{Q}_{n}$.

If $n$ is even, then $\mathcal{L}_{n}$ is, up to isomorphism, the only $n$-dimensional graded filiform Lie algebra.

The preceding matricial presentation of $\mathcal{L}_{n}$ and $\mathcal{Q}_{n}$ shows that these algebras admit a faithful representation of degree the dimension of the algebra.

\section{Control system on graded nilpotent Lie groups}

\subsection{Linear representation of the Lie group $Q_{n}$}

From Vergne's theorem, without loss of generality we can restrict ourselves to consider the classes of nonlinear systems involving the matrix Lie groups $L_{n}$ and $Q_{n}$ associated to the Lie algebras $\mathcal{L}_{n}$ and $\mathcal{Q}_{n}$. The case $L_{n}$, considered in the introduction (corresponding to a car with trailers) has been studied in [S.L]. The system has the canonical form (1).

Let us consider now the linear representation of the Lie algebra $\mathcal{Q}_{n}$ given in the previous section. Taking the exponential of this matrix, we 
find the linear representation of the connected and simply-connected Lie group $Q_{n}$ associated to $\mathcal{Q}_{n}$

$$
\left(\begin{array}{llllllllll}
1 & & & & & & & & & \\
x_{2} & 1 & & & & & & & & \\
x_{3} & x_{1} & 1 & & & & & & & \\
x_{4} & \frac{\left(x_{1}\right)^{2}}{2} & x_{1} & 1 & & & & & & \\
\vdots & \vdots & & \ddots & \ddots & & & & & \\
x_{i} & \vdots & \frac{\left(x_{1}\right)^{i}}{i !} & \cdots & x_{1} & \ddots & & & & \\
\vdots & \vdots & & & & \ddots & \ddots & & & \\
\vdots & \vdots & & & & & \ddots & 1 & & \\
x_{n-1} & \frac{\left(x_{1}\right)^{n-3}}{(n-3) !} & \cdots & \ldots & \ldots & \cdots & \cdots & x_{1} & 1 & \\
x_{n} & y_{n-1} & \cdots & \cdots & \cdots & \cdots & \cdots & y_{3} & x_{1}+x_{2} & 1
\end{array}\right)
$$

where $y_{i}$ are polynomial functions of $x_{1}, \ldots, x_{i}$.

\subsection{Controlled system associated to $\mathcal{Q}_{n}$}

Let us consider the following non linear system

$$
(2):\left\{\begin{array}{l}
\dot{x}_{1}=u_{1}(t) \\
\dot{x}_{2}=u_{2}(t) \\
\dot{x}_{3}=x_{2} u_{1}(t) \\
\dot{x}_{4}=x_{3} u_{1}(t) \\
\vdots \\
\bullet \\
x_{n-1}=x_{n-2} u_{1}(t) \\
\dot{x}_{n}=x_{n-1}\left(u_{1}(t)+u_{2}(t)\right)
\end{array}\right.
$$

Proposition 1. The system (2) can be written as

$$
\left.\dot{X}(t)=\left[u_{1}(t) B_{1}+u_{2}(t) B_{2}\right)\right] X(t)
$$

where $B_{1}$ and $B_{2}$ are the matrices corresponding to the generators of the Lie algebra $\mathcal{Q}_{n}$. 
Proof. Let be

$$
B_{1}:\left(\begin{array}{cccccc}
0 & \cdots & \cdots & \cdots & \cdots & 0 \\
0 & 0 & & & & \vdots \\
0 & 1 & 0 & & & \vdots \\
\vdots & \ddots & \ddots & \ddots & & \vdots \\
\vdots & 0 & \ddots & \ddots & 0 & \vdots \\
0 & \cdots & \cdots & 0 & 1 & 0
\end{array}\right), B_{2}:\left(\begin{array}{cccccc}
0 & \cdots & \cdots & \cdots & \cdots & 0 \\
1 & 0 & & & & \vdots \\
0 & 0 & 0 & & & \vdots \\
\vdots & \ddots & \ddots & \ddots & & \vdots \\
\vdots & 0 & \ddots & 0 & 0 & \vdots \\
0 & \cdots & \cdots & 0 & 1 & 0
\end{array}\right)
$$

These matrices generate the Lie algebra $\mathcal{Q}_{n}$. In fact, if we put

$$
B_{i}=\left[B_{1}, B_{i-1}\right]=B_{1} B_{i-1}-B_{i-1} B_{1}
$$

for $i=3, \ldots, n$ then we also have

$$
\left[B_{i}, B_{n-i+1}\right]=(-1)^{i} 2 B_{n}
$$

for $i=2, \ldots, p=n / 2$. This corresponds to the brackets of $\mathcal{Q}_{n}$. Then we can identify $\mathcal{Q}_{n}$ with the Lie algebra of the matrices $B_{i}$ and the Lie group $Q_{n}$ associated to $\mathcal{Q}_{n}$ is the linear group :

$$
Q_{n}=\left(\begin{array}{llllllllll}
1 & & & & & & & & & \\
x_{2} & 1 & & & & & & & \\
x_{3} & x_{1} & 1 & & & & & & \\
x_{4} & \frac{\left(x_{1}\right)^{2}}{2} & x_{1} & 1 & & & & & \\
\vdots & \vdots & & \ddots & \ddots & & & & \\
x_{i} & \vdots & \frac{\left(x_{1}\right)^{i}}{i !} & \cdots & x_{1} & \ddots & & & \\
\vdots & \vdots & & & & \ddots & \ddots & & \\
\vdots & \vdots & & & & & \ddots & 1 & \\
x_{n-1} & \frac{\left.\left(x_{1}\right)^{n-3}\right) !}{(n-3) !} & \cdots & \cdots & \cdots & \cdots & \cdots & x_{1} & 1 & \\
x_{n} & y_{n-1} & \cdots & \cdots & \cdots & \cdots & \cdots & y_{3} & x_{1}+x_{2} & 1
\end{array}\right)
$$

Thus we have

$$
\left(u_{1}(t) B_{1}+u_{2}(t) B_{2}\right)(X(t))=
$$




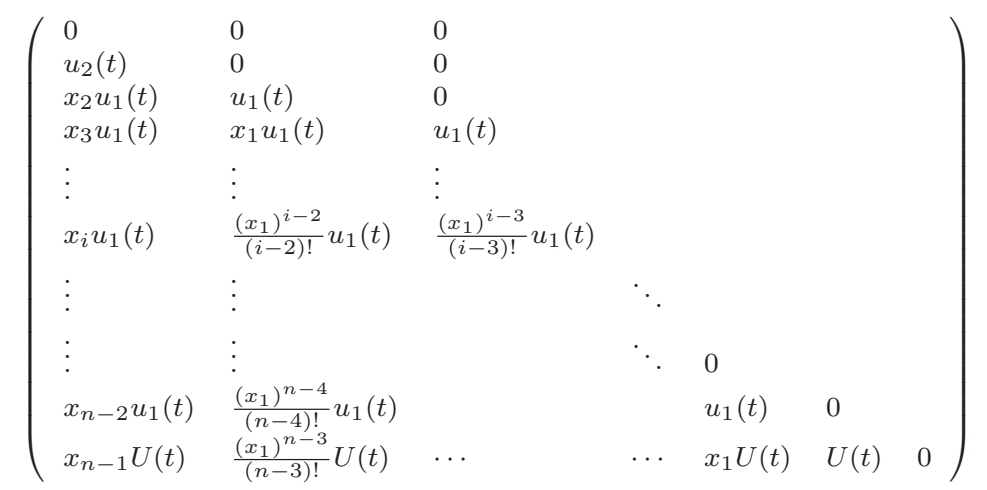

with $U(t)=u_{1}(t)+u_{2}(t)$. This gives the required system.

Theorem 2. The system (2) is controlable.

Recall that the system is controlable if, given two distincts points $X_{0}$ and $X_{f}$ in $\mathcal{Q}_{n}$, there is a finite time $T$ and a function control $u(t)=$ $\left(u_{1}(t), u_{2}(t)\right)$ such that the solution satisfies $X(0)=X_{0}$ and $X(T)=X_{f}$. From [S.L] , such a system is controlable if and only if the matrices $B_{1}$ and $B_{2}$ generate $\mathcal{Q}_{n}$. From the definition of these matrices, $B_{1}, B_{2} \in$ $\mathcal{Q}_{n}-\left[\mathcal{Q}_{n}, \mathcal{Q}_{n}\right]$ and generate the Lie algebra $\mathcal{Q}_{n}$.

\section{The system (2) as a perturbation of (1)}

Let $\varepsilon \in \mathbb{C}$ and consider the linear isomorphism

$$
f_{\varepsilon}: \mathcal{Q}_{n} \rightarrow \mathcal{Q}_{n}
$$

given by $f_{\varepsilon}\left(X_{1}\right)=X_{1}, f_{\varepsilon}\left(X_{i}\right)=\varepsilon X_{i}$ for $i=2, \ldots, n$. If we put $Y_{i}=$ $f_{\varepsilon}\left(X_{i}\right)$, the bracket of $\mathcal{Q}_{n}$ in the basis $\left\{Y_{1}, \ldots, Y_{n}\right\}$ is defined by

$$
\left\{\begin{array}{l}
{\left[Y_{1}, Y_{i}\right]=Y_{i+1}, \quad i=2, \ldots, n-1} \\
{\left[Y_{2}, Y_{n-1}\right]=2 \varepsilon Y_{n}} \\
\vdots \\
{\left[Y_{p}, Y_{p+1}\right]=(-1)^{p} 2 \varepsilon Y_{n}}
\end{array}\right.
$$

Observe that if $\varepsilon$ tends to 0 , the brackets of $\mathcal{Q}_{n}$ tend to those of $\mathcal{L}_{n}$ :

$$
\left\{\left[Y_{1}, Y_{i}\right]=Y_{i+1}, \quad i=2, \ldots, n-1 .\right.
$$


the other bracket beeing nul. This proves that $\mathcal{Q}_{n}$ is a deformation of $\mathcal{L}_{n}$, or that $\mathcal{L}_{n}$ is a contraction of $\mathcal{Q}_{n}$. In this way we can follow the representation of $\mathcal{Q}_{n}$ and see the system (2) as a perturbation of the system (1). Let us consider the representation of $\mathcal{Q}_{n}$ given by the matrices

$$
B^{\varepsilon}=\left(\begin{array}{lllllllll}
0 & & & & & & & \\
a_{2} & 0 & & & & & & & \\
a_{3} & a_{1} & \ddots & & & 0 & & \\
\vdots & & a_{1} & \ddots & & & & \\
a_{i} & & & \ddots & \ddots & & & \\
\vdots & & & & \ddots & \ddots & & \\
a_{n-1} & & & & & & a_{1} & 0 & \\
a_{n} & -\varepsilon a_{n-1} & \ldots & (-1)^{i} \varepsilon a_{i} & \ldots & -\varepsilon a_{3} & a_{1}+\varepsilon a_{2} & 0
\end{array}\right)
$$

If $B_{i}^{\varepsilon}$ is the matrix defined by $a_{i}=1, a_{j}=0$ for $j \neq i$, then we have

$$
\left\{\begin{array}{l}
{\left[B_{1}^{\varepsilon}, B_{i}^{\varepsilon}\right]=B_{i+1}^{\varepsilon}, \quad i=2, \ldots, n-1} \\
{\left[B_{2}^{\varepsilon}, B_{n-1}^{\varepsilon}\right]=2 \varepsilon B_{n}^{\varepsilon}} \\
\vdots \\
{\left[B_{p}^{\varepsilon}, B_{p+1}^{\varepsilon}\right]=(-1)^{p} 2 \varepsilon B_{n}^{\varepsilon}}
\end{array}\right.
$$

that is, the brackets on the new basis. But

$$
\lim _{\varepsilon \rightarrow 0} B_{\varepsilon}=\left(\begin{array}{llllllll}
0 & \cdots & \ldots & \ldots & \ldots & \ldots & \cdots & 0 \\
a_{2} & 0 & & & & & & \vdots \\
a_{3} & a_{1} & \ddots & & & 0 & & \vdots \\
\vdots & & a_{1} & \ddots & & & & \vdots \\
a_{i} & & & \ddots & \ddots & & & \vdots \\
\vdots & & & & \ddots & \ddots & & \vdots \\
a_{n-1} & & & & & a_{1} & 0 & \vdots \\
a_{n} & 0 & \ldots & 0 & \ldots & 0 & a_{1} & 0
\end{array}\right) .
$$

These matrices correspond to the linear representation of $\mathcal{L}_{n}$ given before. 
The nonlinear matrix system

$$
\left.\dot{X}(t)=\left[u_{1}(t) B_{1}^{\varepsilon}+u_{2}(t) B_{2}^{\varepsilon}\right)\right] X(t)
$$

is written :

$$
(3) \quad\left\{\begin{array}{l}
\dot{x_{1}}=u_{1} \\
\dot{x_{2}}=u_{2} \\
\dot{x_{3}}=x_{2} u_{1} \\
\dot{x_{4}}=x_{3} u_{1} \\
\vdots \\
x_{n-1}=x_{n-2} u_{1} \\
\dot{x_{n}}=x_{n-1} u_{1}+\varepsilon x_{n-1} u_{2}
\end{array}\right.
$$

This system is a perturbation of the nonlinear matrix system associated to $\mathcal{L}_{n}$. In fact, if $\varepsilon \rightarrow 0$, we find again the equations of (1). It is clear that the systems (2) and (3) are isomorphic, as they are defined by equivalent representations of $\mathcal{Q}_{n}$.

We can interprete these equations by saying that the last trailer has a perturbation given by the term $\varepsilon x_{n-1} u_{2}$. This is natural, because the role of the first trailer is not the same as that of the last one.

\subsection{Determination of the solutions}

Recall that we can give a global solution of a matrix system associated to a nilpotent Lie algebra by

$$
X(t)=e^{g_{1}(t) A_{1}} e^{g_{2}(t) A_{2}} \ldots e^{g_{n}(t) A_{n}}
$$

where the matrices $A_{i}$ are the elements of the Lie algebra. 


\subsubsection{Solution of (1)}

A direct computation of $X(t)=e^{g_{1}(t) A_{1}} e^{g_{2}(t) A_{2}} \ldots e^{g_{n}(t) A_{n}}$ gives :

$$
\left\{\begin{array}{l}
x_{1}=g_{1} \\
x_{2}=g_{2} \\
x_{3}=g_{1} g_{2}+g_{3} \\
x_{4}=\frac{g_{1}^{2}}{2 !} g_{2}+g_{1} g_{3}+g_{4} \\
\vdots \\
x_{n}=\frac{g_{1}^{n-2}}{(n-2) !} g_{2}+\ldots+g_{n}
\end{array}\right.
$$

The functions $g_{i}$ depends on the control functions $u_{1}$ and $u_{2}$. These relations are defined comparing the derivates of the previous solutions and the equations of (1). We obtain :

$$
\left\{\begin{array}{l}
\dot{g}_{1}=u_{1} \\
\dot{g}_{2}=u_{2} \\
\dot{g}_{3}=-g_{1} \dot{g}_{2} \\
\vdots \\
\dot{g}_{i}=(-1)^{i} \frac{g_{1}^{i-2}}{(i-2) !} \dot{g}_{2} \\
\vdots \\
\dot{g}_{n}=\frac{g_{1}^{n-2}}{(n-2) !} \dot{g}_{2}
\end{array}\right\}
$$

By quadrature, we obtain the expressions of the $g_{i}$.

\subsubsection{Solutions of the system (2)}

The same calculations for the system (2) give:

$$
\left\{\begin{array}{l}
x_{1}=g_{1} \\
x_{2}=g_{2} \\
x_{3}=g_{1} g_{2}+g_{3} \\
x_{4}=\frac{g_{1}^{2}}{2 !} g_{2}+g_{1} g_{3}+g_{4} \\
\vdots \\
x_{n}=\frac{g_{1}^{n-2}}{(n-2) !} g_{2}+\ldots+g_{n}
\end{array}\right.
$$


The relations between the functions $g_{i}$ and the control functions are given by :

$$
\left\{\begin{array}{l}
\dot{\bullet}_{1}=u_{1} \\
\dot{g}_{2}=u_{2} \\
\dot{g}_{3}=g_{1} \dot{g}_{2} \\
\quad \vdots \\
\dot{g}_{i}=(-1)^{i} \frac{g_{1}^{i-2}}{(i-2) !} \dot{g}_{2} \\
\quad \vdots \\
\stackrel{\bullet}{g_{n-1}}=\frac{-g_{1}^{n-3}}{(n-3) !} \dot{g}_{2} \\
\dot{g}_{n}=\frac{g_{1}^{n-2}}{(n-2) !} \dot{g}_{2}+\dot{g}_{2}\left(\frac{g_{1}^{n-3}}{(n-3) !} g_{2}+\frac{g_{1}^{n-4}}{(n-4) !} g_{3}+\ldots+g_{n-1}\right)
\end{array}\right.
$$

\subsubsection{Solutions of the perturbed system (3)}

The link between (1) and (2) is given by solving (3). We obtain :

$$
\left\{\begin{array}{l}
x_{1}=g_{1} \\
x_{2}=g_{2} \\
x_{3}=g_{1} g_{2}+g_{3} \\
x_{4}=\frac{g_{1}^{2}}{2 !} g_{2}+g_{1} g_{3}+g_{4} \\
\vdots \\
x_{n}=\frac{g_{1}^{n-2}}{(n-2) !} g_{2}+\ldots+g_{n}
\end{array}\right.
$$

and find again the same expression as in (2). On the other hand, the perturbation can be read from the relations between the $g_{i}$ and the control functions $u_{i}$ : 


$$
\left\{\begin{array}{l}
\dot{g}_{1}=u_{1} \\
\dot{g}_{2}=u_{2} \\
\dot{g}_{3}=-g_{1} \dot{g}_{2} \\
\vdots \\
\dot{g}_{i}=(-1)^{i} \frac{g_{1}^{i-2}}{(i-2) !} \dot{g}_{2} \\
\vdots \\
g_{n-1}=\frac{-g_{1}^{n-3}}{(n-3) !} \dot{g}_{2} \\
\dot{g}_{n}=\frac{g_{1}^{n-2}}{(n-2) !} \dot{g}_{2}+\varepsilon \dot{g}_{2}\left(\frac{g_{1}^{n-3}}{(n-3) !} g_{2}+\frac{g_{1}^{n-4}}{(n-4) !} g_{3}+\ldots+g_{n-1}\right)
\end{array}\right.
$$

When $\varepsilon \rightarrow 0$, we find the expressions of the $g_{i}$ of the system (1).

\section{References}

[A] Ancochea Bermúdez J.M., On the rigidity of solvable Lie algebras. In Deformation Theory of Algebras and Structures and Applications. Hazewinkel Ed. Kluwer Acad. Publis. 403-445, 1988.

[A.C] Ancochea Bermúdez J.M.,; Campoamor Strusberg O., On certain families of naturally graded Lie algebras, to appear in J. Pure Appl. Algebra.

[G.K] Goze M; Khakimdjanov Y., Nilpotent Lie Algebras. Kluwer Academic Publishers, MIA 361, 336p, 1996.

[R] Remm E., Systèmes controlables sur les groupes de Lie. mémoire DEA. Université de Haute Alsace. Mulhouse, 1997.

[S.L] Silva Leite F., Control Systems on matrix Lie groups, Actas/Proceedings EAMA-97. 295-306, 1997

[V] Vergne M., Cohomologie des algèbres de Lie nilpotentes. Bull. Soc. Math. France, 98, 81-116, 1970.

Laboratoire de Mathématiques

4, rue des Frères Lumière

Recibido: 22 de Enero de 2001

F. 68093 Mulhouse Cedex

Revisado: 22 de Marzo de 2001 